This item was submitted to Loughborough's Research Repository by the author.

Items in Figshare are protected by copyright, with all rights reserved, unless otherwise indicated.

\title{
Electrospun polycaprolactone nano-fibers supports growth of human mesenchymal stem cells
}

\section{PLEASE CITE THE PUBLISHED VERSION}

http://dx.doi.org/10.1109/NANO.2013.6720851

PUBLISHER

(C) IEEE

VERSION

AM (Accepted Manuscript)

LICENCE

CC BY-NC-ND 4.0

\section{REPOSITORY RECORD}

Fotticchia, Andrea, Yang Liu, Emrah Demirci, and Cristina Lenardi. 2019. "Electrospun Polycaprolactone Nano-fibers Supports Growth of Human Mesenchymal Stem Cells”. figshare.

https://hdl.handle.net/2134/14023. 
This item was submitted to Loughborough's Institutional Repository (https://dspace.lboro.ac.uk/) by the author and is made available under the following Creative Commons Licence conditions.

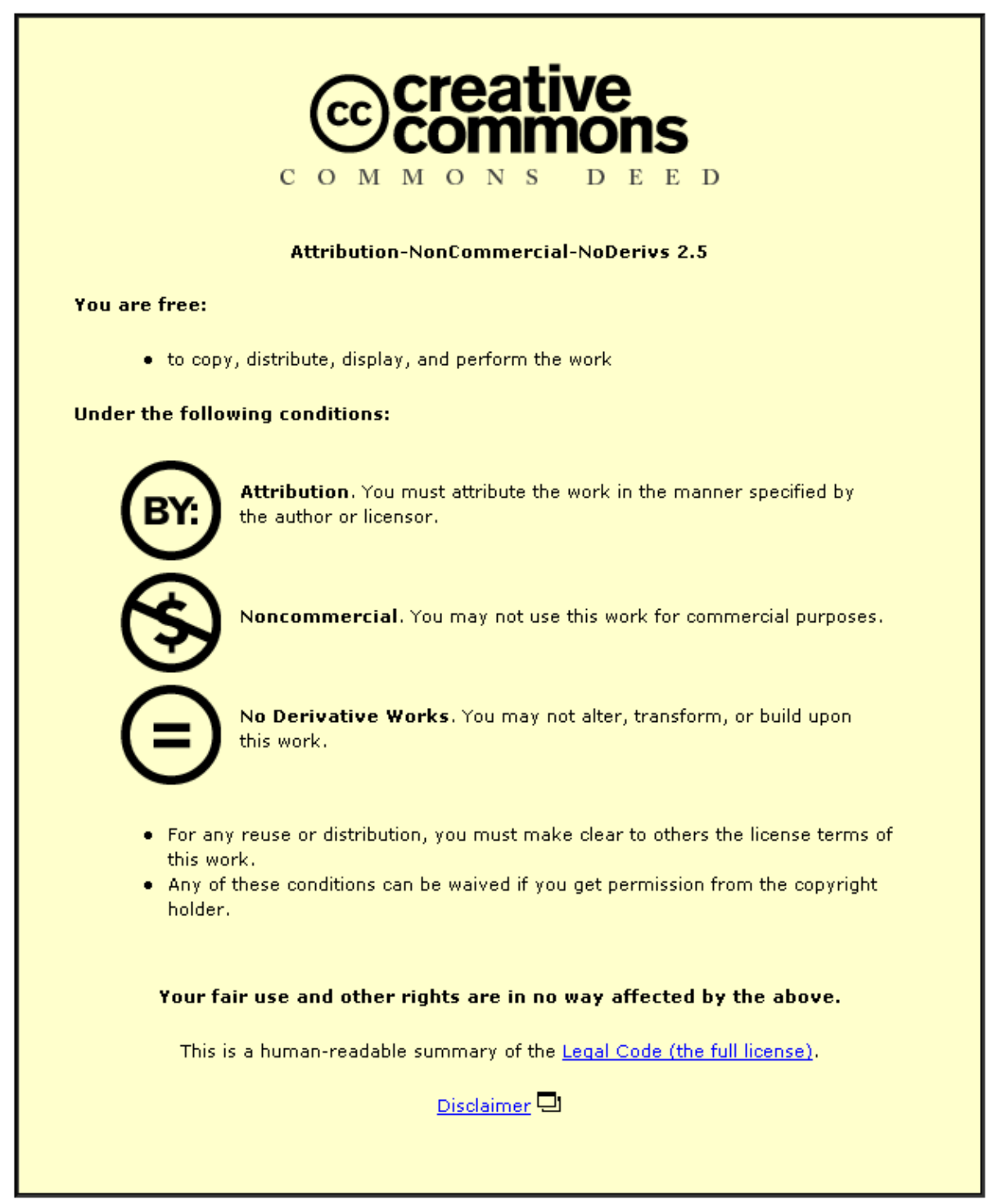

For the full text of this licence, please go to: http://creativecommons.org/licenses/by-nc-nd/2.5/ 


\title{
Electrospun Polycaprolactone Nano-fibers Supports Growth of Human Mesenchymal Stem Cells
}

\author{
Andrea Fotticchia, Yang Liu, Emrah Demirci and Cristina Lenardi
}

\begin{abstract}
Electrospun polycaprolactone (PCL) layers with sub-micron fibres arranged in random, semi-aligned and aligned manners were prepared and their anisotropic mechanical property were further characterised at various stretching angle. Proof of principle study was carried out to assess biocompatibility of electrospun layers with human mesenchymal stem cells (hMSCs). Cell adhesion, viability and orientation outcomes showed that aligned PCL nano-fibers can be applied as a template to guide the alignment of hMSCs and could be a promising scaffold for tissue engineering of anisotropic annulus fibrosus in spinal inter-vertebral disc.
\end{abstract}

\section{INTRODUCTION}

Nowadays low back pain due to spinal inter-vertebral disc (IVD) degeneration is one of the major concerns for national health systems, causing the largest number of inactivity events in under 45 years old people of USA [1]. The annulus fibrosus (AF) within IVD is multi-layered tissue and its rupture as the consequence of IVD degeneration can lead to disc bulging and herniation, resulting in low back pain [2]. AF is composed of stacked lamellae with collagen fibers oriented at $\pm 30^{\circ}$ relative to the adjacent layer [3]. As an avascular tissue, injured AF cannot be self-repaired [4]. Thus technology of tissue engineered AF using electrospun nanofibres with controlled fiber arrangements could supply a template allowing cell alignment and deposition of oriented collagen fiber, hence, replacing damaged portions of the IVD.

Up to date electrospinning has shown its versatility as it was exploited in several scientific fields to produce fibers with many different features at nano-scale [5] and a wide range of polymers belonging to different classes have been electrospun [6]. The most common types include polyesters, such as polylactic acid, polyglycolic acid and polycaprolactone (PCL), because of their commercial availability, ease in processing and well known biocompatibility [7]. In the current work polycaprolactone non-woven layers with different fiber arrangement were fabricated. Growth of human mesenchymal stem cells

*Research supported by Loughborough University graduate school PhD studentship (AF) and Royal Society.

Y. Liu is with the Mechanical and Manufacturing Engineering department, Loughborough University, LE11 3TU, UK (e-mail: y.liu3@lboro.ac.uk).

C. Lenardi is with Fondazione Filarete and Dipartimento di Fisica, Universita' di Milano, Via Celoria 16, 20133 Milano (e-mail: cristina.lenardi@mi.infn.it).

E. Demirci is with the Mechanical and Manufacturing Engineering department, Loughborough University, LE11 3TU, UK (e-mail: e.demirci@lboro.ac.uk).

A. Fotticchia is with the Mechanical and Manufacturing Engineering department, Loughborough University, LE11 3TU, UK (0044 (0)1509564893; e-mail: a.fotticchia@lboro.ac.uk).
(hMSCs) on the said layers was evaluated further in order to correlate the features of fiber arrangement with cell alignment.

\section{MATERIALS AND METHODS}

\section{A. Materials}

Polycaprolactone (Mn=70,000-90,000) and corresponding solvents, including tetrahydrofuran (THF), N,N-dimethylformamide (DMF), chloroform (CF) and formalin, were supplied by Sigma-Aldrich. Cell culture reagents were all purchased from Lonza except antibiotic and antimycotic from Life Technologies.

\section{B. Electrospun layer fabrication}

11 and 15\% wt/wt PCL solution were prepared in the following solvent mixtures: THF+DMF (1:1) or CF+DMF (1:1). The whole fabrication system, including machine (Ske s.r.l., Milano), syringe pump (NE-1010, New Era Pump Systems), syringes (Terumo) of different size, flexible tube (z609706 Bohlender, Sigma-Aldrich) and metal spinneret (inner diameter $=1 \mathrm{~mm}$ ), was placed in a chemical fume hood inside a clean room (Class 8, ISO 14644). The tension between the syringe nozzle and the collector was given by a 16-18 kV potential difference through a power supply (SL150, Spellman). Prior to electrospinning the drum collector was wrapped with aluminum foil to facilitate the layer removal at the end of the process. The solution was kept spinning continuously by controlling the following parameters: $16 \mathrm{~cm}$ spinneret to collector distance, $3 \mathrm{ml} / \mathrm{h}$ flow rate and 60-3000 rpm collector speed. Layers with three different types of fiber arrangement, random, semi-aligned and aligned fibers, were fabricated by tuning the collector at velocities of 60, 2000 and $3000 \mathrm{rpm}$.

\section{Morphology, thickness, fibers orientation and mechanical properties of PCL layers}

The PCL nanofibres on the electrospun layers were gold sputter coated (Polaron 5100) and analyzed with the electron microscope (Zeiss Sigma FE). Fiber diameter and orientation degree shown in electron microscope images (Fig. 1) were quantified further using Image-J software. Fiber diameter was calculated by measuring 30 randomly chosen fibers on three locations per layer. Images at lower magnification were used to assess fiber orientation through 50 random measurements per sample. Orientation was evaluated by measuring the angles between each fiber and a specific direction chosen as reference. In case of fibres that did not clearly follow a specific direction but curled with coils, a random portion of them was chosen. Average and standard deviation were calculated with the aid of Microsoft Excel 2010. 

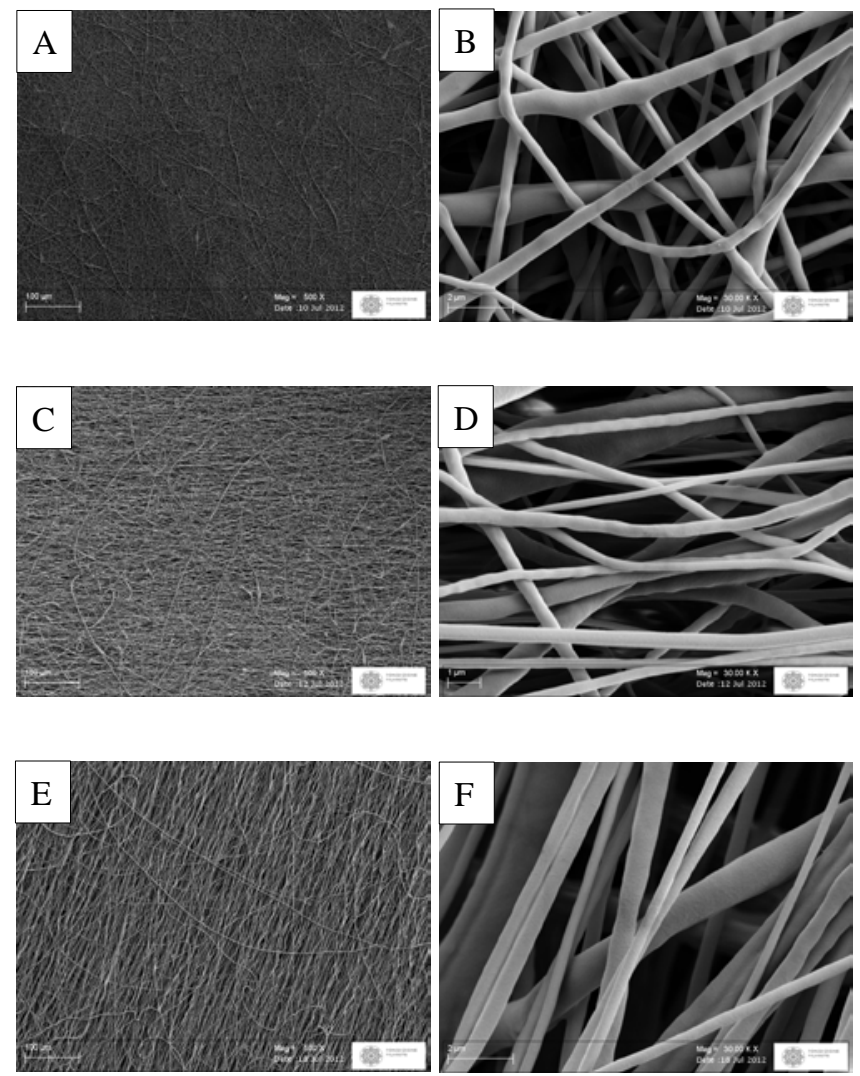

Figure 1. Comparison between layers made of fibers with approximately the same diameter. From the top to the bottom: random ( $A$ and $B)$, semi-aligned $(C$ and $D)$ and aligned fibers ( $E$ and F). Left column: 500x magnification, scale bar $100 \mu \mathrm{m}$; right column: $30 \mathrm{kx}$ magnification, scale bar $2 \mu \mathrm{m}$.

To quantify thickness of PCL layers, for each sample three electrospun strips of approximately $10 \mathrm{~mm}$ width were analysed with a non-contact optical profilometer (Talysurf CLI 2000, Taylor Hobson). Each strip was scanned along the width and the profile analysed with the aid of the supplier software (TalyMap Platinum V. 5.0.4) to obtain the average height.

Mechanical properties of the different fiber arrangements were evaluated by using MicroTester 5848 (Instron) equipped with a $5 \mathrm{~N}$ load cell. Specimens of $10 \mathrm{~mm}$ x 50 $\mathrm{mm}$ were prepared and tested in triplicate with $0.1 \mathrm{~N}$ preload, at a constant stretching rate of $5 \mathrm{~mm} / \mathrm{min}$. The stress-strain curve obtained was not all linear but two different linear regions could be identified for each sample before breakage. Young's modulus was evaluated as $E_{\text {low }}$ and $\mathrm{E}_{\mathrm{high}}$, according to two different linear regions of stress strain curve per sample.

\section{Cell culture}

Prior to seeding of hMSC electrospun layers were sterilized by exposure to 30 mins of UV light per side. Bone marrow human mesenchymal stem cells (Lonza) were cultured onto layers with random, semi-aligned and aligned PCL fibres. Since after fabrication some of the layers presented a wavy surface, samples of PCL layers were mounted onto Cell Crown (Sigma-Aldrich) devices in order to flatten the samples and facilitate cell seeding procedure and microscopy analysis. Cell Crowns were, hence, blocked inside 12 multiwell plates with samples soaked in culture medium for 24 hours before cell seeding. Cell culture medium was prepared as follows: $87 \%$ DMEM, 10\% Foetal bovine serum, $1 \%$ non-essential amino acids, $1 \%$ Lglutamine and $1 \%$ antibiotic and antimycotic. $30 \mu \mathrm{l}$ suspension of passage 6 human mesenchymal stem cells were seeded at $5.5 \times 10^{4}$ cells/sample and the seeded samples were incubated for 2 hrs followed by topping up 2 $\mathrm{ml}$ of cell culture medium.

During 20 days of culture, cell viability was assessed on day 1 and also every 5 days using Alamar Blue assay (Life technologies). At each time point culture medium was removed and specimens were rinsed in PBS three times. 2 $\mathrm{ml}$ medium with $10 \%$ Alamar blue were pipetted into each well. After 4 hours incubation, $100 \mu$ l aliquots of alamar blue solution were analyzed with a fluorescent plate reader (FLUOstar Omega, BMG Labtech). At the end of 20 days cells were fixed and double stained with Phalloidin Alexa Fluor-555 (Life technologies) and Hoechst 33342 (Invitrogen) to evaluate Actin filaments and nuclei respectively.

\section{RESULTS}

\section{A. Effect of solvent and process parameters on morphology}

The electrospinning machine parameters were fine-tuned in order to fabricate matrices with different feature combinations, namely layer thickness, fiber diameter and alignment degree. Fiber diameter was found to be affected mostly by the solution concentration when solvent system used were controlled. Table I clearly shows that fiber diameter is proportional to the polymer concentration. Concentrations above $14 \%$ made the fibers reach diameters of $1 \mu \mathrm{m}$ or beyond. The other process parameters did not vary considerably from one configuration to another and were modified in order to make the fabrication proceed smoothly. Fig. 1 shows micrographs taken from samples with random (Fig. 1. A and B), semi-aligned (Fig. 1. C and D) and aligned (Fig. 1. E and F) fibers having an average diameter of 400$600 \mathrm{~nm}$. The layer with random fibers (Fig. 1. A) shows the presence of some spheres and fibers at low magnification. Most of fibers on aligned and semi-aligned layers are straight (Fig. 1. D and E) but few of them are wavy as observed at lower magnification (Fig. 1. C and E). Furthermore at low magnification the presence of these wavy fibers out of the main fiber direction was mainly observed on the top of the matrix (Fig. 1. C and E). These fibers forming a significant different angle compared to the main orientation can be seen in larger amount on semi-aligned samples. At high magnification all the fibers are characterized by a smooth surface morphology and are flawless at the nanometric scale.

TABLE I. Comparison between different polymer concentrations used and the relative fibre diameter.

\begin{tabular}{|c|l|l|l|}
\hline $\begin{array}{c}\text { PCL } \\
\text { concentration } \\
{[\% \text { wt/wt] }}\end{array}$ & Solvent system & $\begin{array}{c}\text { Diameter } \pm \\
\text { Standard deviation } \\
{[\boldsymbol{\mu m}]}\end{array}$ & $\begin{array}{c}\text { Type of } \\
\text { sample }\end{array}$ \\
\hline $11 \%$ & CF+DMF 1:1 & $0.40 \pm 0.15$ & Random \\
\hline $14 \%$ & THF+DMF 1:1 & $1.17 \pm 0.57$ & Random \\
\hline $15 \%$ & THF+DMF 1:1 & $1.67 \pm 0.48$ & Random \\
\hline
\end{tabular}




\begin{tabular}{|l|l|l|l|}
\hline $11 \%$ & CF+DMF 1:1 & $0.40 \pm 0.14$ & $\begin{array}{l}\text { Semi- } \\
\text { aligned }\end{array}$ \\
\hline $14 \%$ & THF+DMF 1:1 & $0.95 \pm 0.49$ & $\begin{array}{l}\text { Semi- } \\
\text { aligned }\end{array}$ \\
\hline
\end{tabular}

B. Thickness, fiber orientation and mechanical properties of PCL layers

The thickness of PCL layers was between 0.40 and $0.60 \mu \mathrm{m}$. Fig. 2 reveals the distribution of angles between single fiber and the main fiber direction for layers of semi-aligned (left) and aligned (right) PCL fibers. Distribution of angle on semi-aligned samples was broader than on aligned sample as shown by the histogram and the standard deviation (41.48 for semi-aligned layers and $29.03^{\circ}$ for aligned layers). In the former, a few fibers perpendicular to the main direction $\left(90^{\circ}\right.$ angle) were also detected whilst in the latter no perpendicular fiber was found. Moreover $46 \%$ and $64 \%$ of the fibres of semi-aligned and aligned layers were identified with angle in a range of $\pm 15^{\circ}$ around the main direction.

Anisotropic mechanical properties of semi-aligned and aligned layers were measured by changing stretching angle between the tension force applied and the main fiber direction by $0^{\circ}, 30^{\circ}, 60^{\circ}$ and $90^{\circ}$. Young's moduli of the said specimens are summarized in Fig. 3. When stretched in parallel to the predominant fiber direction $\left(0^{\circ}\right)$, aligned layers showed a higher Young's modulus $\left(\mathrm{E}_{\mathrm{low} 0}=53.64 \pm 12.26 \mathrm{MPa}\right.$, $\left.E_{\text {high } 0^{0}}=21.42 \pm 7.17 \mathrm{MPa}\right)$ than semi-aligned stretched at the same direction $\left(\mathrm{E}_{\mathrm{low} 0^{0}}=27.94 \pm 11.63 \mathrm{MPa}, \mathrm{E}_{\mathrm{high} 0^{0}}=11.92 \pm 3.81\right.$ $\mathrm{MPa})$ and random fibers $\left(\mathrm{E}_{\text {low }}=2.77 \pm 0.28 \mathrm{MPa}\right.$ and $\mathrm{E}_{\text {high }}=1.25 \pm 0.09 \mathrm{MPa}$ ). Conversely with stretching angle close to $90^{\circ}$ random fiber layers were stiffer than aligned
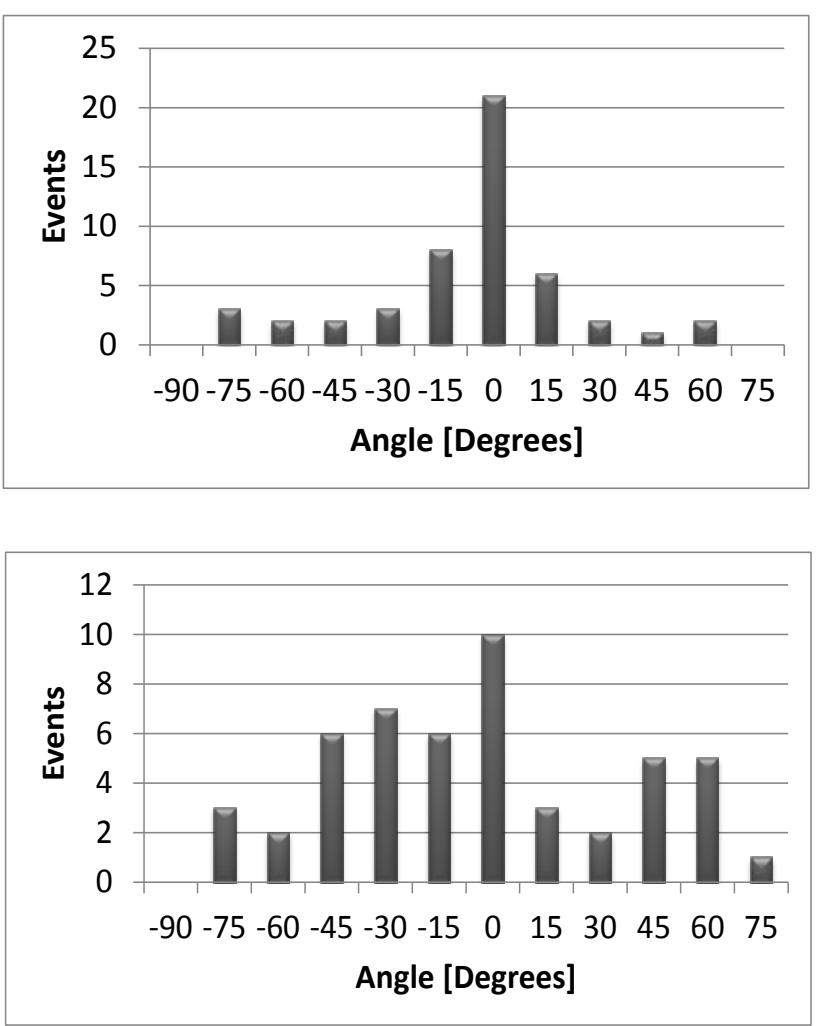

Figure 2. Comparison between fibers angle distributions of aligned (top) and semi-aligned (bottom) layers. On the horizontal axis the values represent the starting angle of the bin. Standard deviations are 41.48 and $29.03^{\circ}$ respectively.

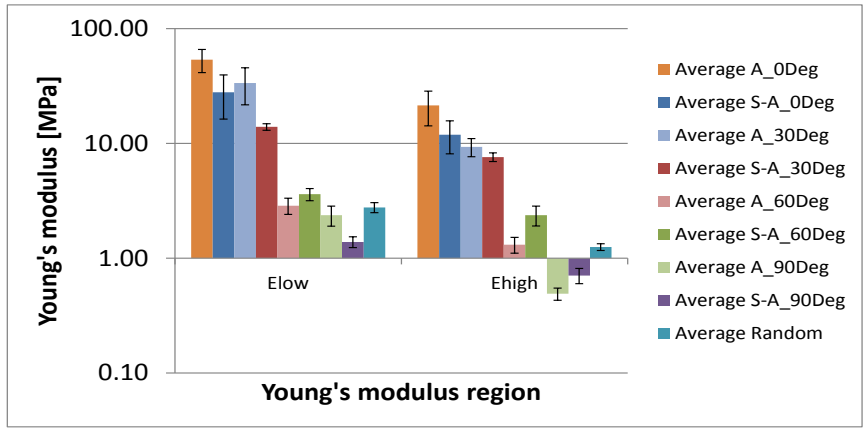

Figure 3. Young's moduli of layers with random, semi-aligned and aligned PCL fibers. Logarithmic scale was used for the vertical axis. Note: A/S-A_nDeg represents Aligned/Semi-aligned fiber prepared and stretched at an angle of $n$ degrees relative to the main fiber orientation.

$\left(E_{\text {low90 }}=2.37 \pm 0.47 \mathrm{MPa}, E_{\text {high } 90^{\circ}}=0.49 \pm 0.06 \mathrm{MPa}\right)$ and semialigned ones $\left(\mathrm{E}_{\mathrm{low} 90^{\circ}}=1.39 \pm 0.15 \mathrm{MPa}, \mathrm{E}_{\mathrm{high} 90^{\circ}}=0.71 \pm 0.11\right.$ MPa), especially at high strain. At 0 and $30^{\circ}$ stretching angles aligned fiber layer showed a higher Young's modulus compared to semi-aligned while the trend was reverted at $60^{\circ}$ and $90^{\circ}$ stretching direction (Fig. 3). Across all the specimens stretched at different angles, the lowest and highest elastic moduli were found on aligned specimens, at $90^{\circ}$ and $0^{\circ}$ stretching respectively.

\section{Growth of hMSC on PCL layers}

hMSC cultured on PCL layers were viable during the 20 days culture with slightly decrease of cell viability at day 10 and 20; nevertheless the decrease has always been found to be not significant compared to day 1 (Fig. 4).

Images of cells double stained for actin filament (red) and nuclei (blue) are shown in Fig. 5. hMSCs formed a monolayer on all the electrospun specimens. On random arranged fibres no specific cell alignment was observed as

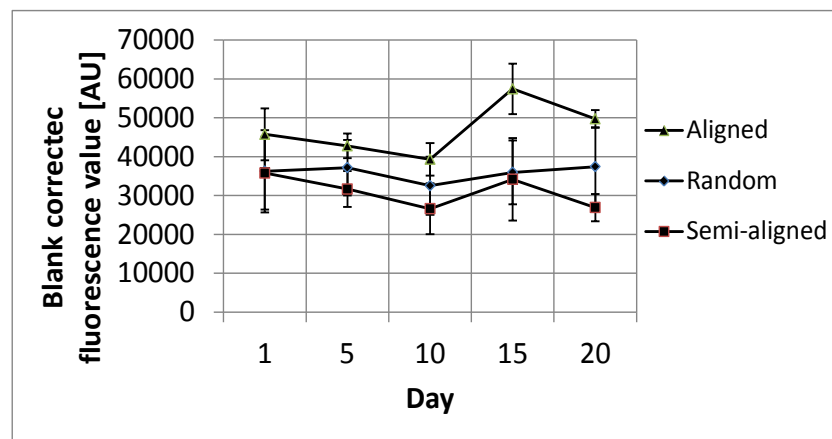

Figure 4. Viability test over a period of 20 days.
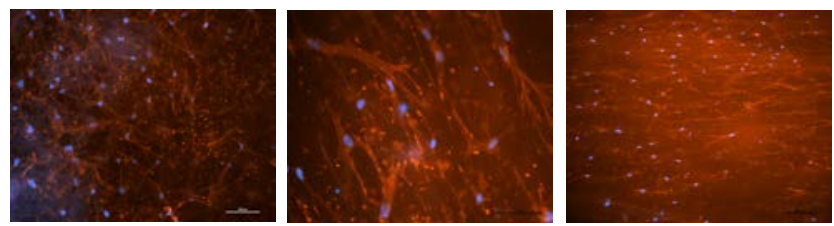

Figure 5. Double stained cells cultured on different type of layers. Phalloidin Alexa Fluor-555 conjugated and Hoechst 33342 dyes were used to identify actin filaments (red) and nuclei (blue) respectively. From the left to right: random, semi-aligned and aligned fibres. Scale bar $100 \mu \mathrm{m}$. 
evidenced by the lack of any ordered arrangement of actin filaments. Some hMSCs assumed an elongated shape, but no obvious direction was noticed. Cell alignment occurred on semi-aligned and aligned specimens, where most of cells attached to the semi-aligned and nearly all cells attached to the aligned ones assumed an elongated shape. Their actin filaments and the long axis of nuclei were aligned with those of adjacent cells and oriented in the same direction.

\section{DISCUSSION}

The aim of this study is to assess the biocompatibility of electrospun PCL matrices and evaluate how their morphological features affect alignment of hMSCs. The electrospinning technique proved to be reliable enough to finely tune the morphological features [8, 9]. By controlling the processing parameters we managed to modify only one characteristic of the PCL layers, thus allowing us to compare the effect of a single feature at a time. Particularly layers with different fiber arrangement were compared while keeping other parameters constant, including fiber diameter and layer thickness. On aligned and semi-aligned layers, still few PCL fibers perpendicular to the main direction were also detected. It is likely that in those cases the polymeric jet, during the fabrication process, had a very high local velocity able to make the collector speed ineffective. Moreover it appeared that the majority of the out of alignment fibers was noticed on the top of the matrix. This could be due to synchronization problems and delay of response when syringe pump was stopped at the end of the fabrication process.

For aligned and semi-aligned electrospun PCL layers, due to the presence of anisotropic structure, their mechanical properties were dependent on the angle of stretching relative to predominant fiber direction. Semi-aligned and in particular aligned electrospun layers were stiffer than random ones when stretched at a $0^{\circ}$ angle. At $90^{\circ}$ the trend was reverted because in this case only the weak entanglement of parallel fibers on semi-aligned and aligned layers was against the load applied. It was most likely that fibers were just separated from each other, rather than broken, by the applied stretching force. This might explain why at $90^{\circ}$ no abrupt rupture was occurred, even till the entire specimen was split into two fragments. The Young's moduli found are quite consistent to those identified by Nerurkar et al. and Koepsell et al. [10, 11]. Moreover, compared to AF tissue benchmarks, the moduli were similar to those identified by Holzapfel et al. on single lamellae but lower than the ranges claimed by Skaggs et al. and Zhu et al. [12-14].

The fabricated electropun PCL layer can support hMSCs growth and semi-aligned and aligned layers could work as a template to guide the orientation of the attached hMSCs, consistent with similar contact guidance experiments published [9, 15], whilst such effects were not observed on random fiber layers. PCL electrospun layers fabricated in the current study were cytocompatible and might support cell growth for a long period of time without the aid of any physical or chemical post-processing treatment.

\section{CONCLUSION}

It was demonstrated that it is feasible to apply electrospun aligned PCL nano-fibers as a guidance of hMSCs orientation. Our future research will concentrate on how such guidance would affect the orientation of collagen fiber deposed by the seeded hMSC, simulating the structure of AF tissue.

\section{ACKNOWLEDGMENT}

We would like to thank supports from Fondazione Filarete for getting access to electrospinning equipment.

\section{REFERENCES}

[1] G. B. Andersson, "Epidemiological features of chronic low-back pain.,” Lancet, vol. 354, no. 9178, pp. 581-5, Aug. 1999.

[2] P. P. Raj, "Intervertebral Disc: Anatomy-PhysiologyPathophysiology-Treatment,” Pain Practice, vol. 8, no. 1, pp. 1844, 2008.

[3] J. L. Bron, M. N. Helder, H.-J. Meisel, B. J. Van Royen, and T. H. Smit, "Repair, regenerative and supportive therapies of the annulus fibrosus: achievements and challenges.," European spine journal, vol. 18, no. 3, pp. 301-13, Mar. 2009.

[4] J. Urban, S. Smith, and J. C. T. Fairbank, "Nutrition of the intervertebral disc.," Spine, vol. 29, no. 23, pp. 2700-9, Dec. 2004.

[5] J. Fang, H. Niu, T. Lin, and X. Wang, “Applications of electrospun nanofibers,” Chinese Science Bulletin, vol. 53, no. 15, pp. 2265-2286, Aug. 2008.

[6] Z. Huang, “A review on polymer nanofibers by electrospinning and their applications in nanocomposites,” Composites Science and Technology, vol. 63, no. 15, pp. 2223-2253, 2003.

[7] S. Agarwal, J. H. Wendorff, and A. Greiner, "Use of electrospinning technique for biomedical applications,” Polymer, vol. 49, no. 26, pp. 5603-5621, Dec. 2008.

[8] G. H. Kim, "Electrospun PCL nanofibers with anisotropic mechanical properties as a biomedical scaffold.," Biomedical materials, vol. 3, no. 2, pp. 1-8, Jun. 2008.

[9] W.-J. Li, R. L. Mauck, J. a Cooper, X. Yuan, and R. S. Tuan, "Engineering controllable anisotropy in electrospun biodegradable nanofibrous scaffolds for musculoskeletal tissue engineering.," Journal of biomechanics, vol. 40, no. 8, pp. 168693, Jan. 2007.

[10] N. L. Nerurkar and D. M. Elliott, "Mechanics of oriented electrospun nanofibrous scaffolds for annulus fibrosus tissue engineering," Journal of orthopaedic, vol. 25, no. 8, pp. 10181028, 2007.

[11] L. Koepsell, T. Remund, J. Bao, D. Neufeld, H. Fong, and Y. Deng, "Tissue engineering of annulus fibrosus using electrospun fibrous scaffolds with aligned polycaprolactone fibers.," Journal of biomedical materials research. Part A, vol. 99, no. 4, pp. 56475, Dec. 2011.

[12] G. a Holzapfel, C. a J. Schulze-Bauer, G. Feigl, and P. Regitnig, "Single lamellar mechanics of the human lumbar anulus fibrosus.," Biomechanics and modeling in mechanobiology, vol. 3, no. 3, pp. 125-40, Mar. 2005.

[13] D. Skaggs, M. Weidenbaum, J. Iatridis, A. Ratcliffe, and V. Mow, "Regional Variation in Tensile Properties and Biochemical Composition of the Human Lumbar Anulus Fibrosus," Spine, vol. 19, no. 12, pp. 1310-1319, 1994.

[14] D. Zhu, G. Gu, W. Wu, H. Gong, W. Zhu, T. Jiang, and Z. Cao, "Micro-structure and mechanical properties of annulus fibrous of the L4-5 and L5-S1 intervertebral discs.," Clinical biomechanics (Bristol, Avon), vol. 23 Suppl 1, pp. S74-82, Jan. 2008.

[15] N. L. Nerurkar, W. M. Han, R. L. Mauck, and D. M. Elliott, "Homologous structure-function relationships between native fibrocartilage and tissue engineered from MSC-seeded nanofibrous scaffolds.," Biomaterials, vol. 32, no. 2, pp. 461-8, Jan. 2011. 